\title{
Optical and magneto-optical properties of thin films for high density information recording
}

\author{
V.G. Kravets ${ }^{1)}$, L.V. Poperenkoo), I.V. Yurgelevich' ${ }^{2)}$, A. K. Petford-Long ${ }^{3)}$ \\ ${ }^{1}$ Institute for Information Recording, 2 Shpak St, 03113 Kyiv, Ukraine \\ ${ }^{2}$ Department of Physics, Taras Shevchenko Kyiv University, 6 Av. Acad. Glushkov, 03022 Kyiv, Ukraine \\ ${ }^{3}$ Department of Materials, University of Oxford, Oxford OXI 3PH, United Kingdom
}

\begin{abstract}
It was found that optical and magneto-optical (MO) properties of thin film materials have potential application for blue laser recording. For this purpose, we have used a combination of ellipsometric and polar MO Kerr effect measurements. The optical and magneto-optical properties of $\mathrm{Mn}\left(\mathrm{Sb}_{1-\mathrm{x}} \mathrm{Bi}_{\mathrm{x}}\right)$ and $\mathrm{Co}-\mathrm{Pt}$ nanocrystalline films were investigated for incident photons in the energy range from 1.3 to $4.0 \mathrm{eV}$. A large Kerr rotation angle of about $0.57^{0}$ was observed at photon energy $\hbar \omega \approx 2.65 \mathrm{eV}$ for the $\mathrm{Mn}_{55} \mathrm{Sb}_{36} \mathrm{Bi}_{9}$ film with an average grain size of $30 \mathrm{~nm}$. The changes of the exchange splitting and the spin-orbit interaction strength are responsible for the strong dependence of Kerr effect in $\mathrm{Mn}\left(\mathrm{Sb}_{1-\mathrm{x}} \mathrm{Bi}_{\mathrm{x}}\right)$ nanocrystalline films. It was shown that the large MO polar Kerr rotation angle is correlated with a perpendicular magnetic anisotropy in nanocrystalline $\mathrm{Co}_{3} \mathrm{Pt}$ films
\end{abstract}

Keywords: optical and magneto-optical properties; Kerr effect; nanocrystalline films.

Paper received 14.03.02; revised manuscript received 23.05.02; accepted for publication 25.06.02.

\section{Introduction}

The magneto-optical recording is based on switching the magnetization direction of perpendicularly magnetized domains in a magnetic thin film. As the magneto-optical (MO) recording technology continues to improve, the search for media with high MO performance at short wavelengths has becomes the focus of many research works. The storage capacity of an optical disk is determined by the size of the laser sport used for writing and reading. In the near future, the storage capacity will be further increased by using shorter wavelength (blue) lasers or objective lenses with a higher numerical aperture, or a combination of both [1].

Amorphous $\mathrm{TbFeCo}$ films and $\mathrm{Co} / \mathrm{Pt}$ multilayers are presently the media of choice for red and infrared MO recording. The Kerr effect of $\mathrm{TbFeCo}$ and $\mathrm{Co} / \mathrm{Pt}$ films, however, decreases at short wavelengths [2]. Thin films of $\mathrm{MnBi}$ or $\mathrm{MnBiX}$ have been studied extensively for development as magneto-optical (MO) storage media because they show a large magneto-optical Kerr effect (MOKE) signal. However, there are problems related to a phase transformation, chemical stability of MnBi films and the grain boundary noise [3]. In order to solve these problems we have proposed to substitute $\mathrm{Bi}$ for $\mathrm{Sb}$ in $\mathrm{MnSb}$ to obtain the compound $\mathrm{Mn}\left(\mathrm{Sb}_{1-\mathrm{x}} \mathrm{Bi}_{\mathrm{x}}\right)$, which possesses better physical and chemical properties compared to $\mathrm{MnBi}$ or $\mathrm{MnSb}$. The $\mathrm{MnSbBi}$ films are promising for the future UV-range MO recording, because of the short-wavelength energy position of their Kerr rotation peak accessible for UV lasers. MnSbBi films are attractive also because Mn, $\mathrm{Sb}$ and Bi metals are lower in price than platinum. Among many different systems, the Co-Pt alloys receive significant attention as high-density MO recording media due to their large perpendicular magnetic anisotropy, and high readout efficiency provided by the reflectivity $\mathrm{R}$ and Kerr rotation $\theta_{\mathrm{K}}$.

In this work, we present the dielectric tensor and polar Kerr effect for several MO media which are considered as candidates for the next generation devices that will operate with blue lasers. The optical and MO properties have been measured in the wavelength region of $310-800 \mathrm{~nm}$, covering the full range of interest for $\mathrm{MO}$ data storage. Adding $\mathrm{Bi}$ into $\mathrm{MnSb}$ and putting the $\mathrm{Sb}$-seed layer on the substrate the Kerr effect was enhanced, showing higher maximum Kerr rotation in the measured frequency region. The film with the configuration of $\mathrm{Mn} / \mathrm{Sb} / \mathrm{Bi}$ shows the maximum Kerr rotation peak around $2.5 \mathrm{eV}$.

\section{Sample preparation and experiments}

Nanocrystalline $\mathrm{Mn}\left(\mathrm{Sb}_{1-\mathrm{x}} \mathrm{Bi}_{\mathrm{x}}\right)$ and $\mathrm{Co}_{\mathrm{x}} \mathrm{Pt}_{1-\mathrm{x}}$ films were grown by alternating evaporation of $\mathrm{Mn}, \mathrm{Bi}$ and $\mathrm{Sb}$ or $\mathrm{Co}(\mathrm{Pt})$ metals in a vacuum to obtain multilayer systems with the $[\mathrm{Sb} / \mathrm{Mn} / \mathrm{Bi}]_{6} / /$ substrate or $[\mathrm{Co} / \mathrm{Pt}]_{6} / /$ substrate configuration. They were prepared by $\mathrm{rf}$ and $\mathrm{dc}$ magnetron sputtering onto glass, mica and silicon 


\section{V.G. Kravets et al.: Optical and magneto-optical properties...}

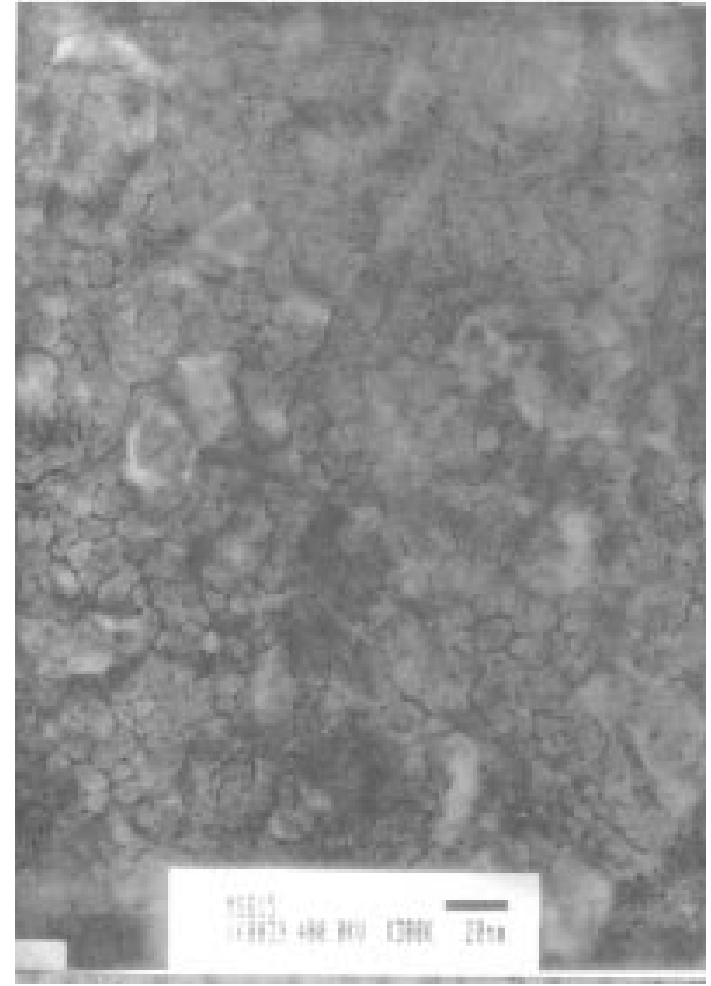

a)

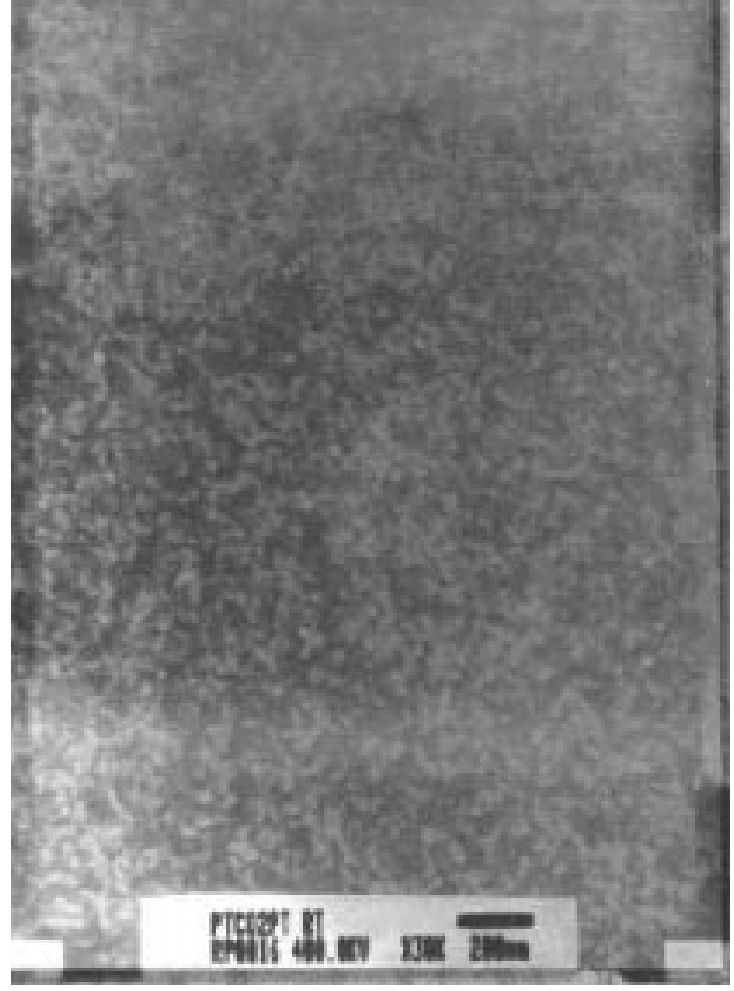

b)

Fig. 1. TEM (bright-field) plan-view image of the nanocrystalline films clearly showing the polycrystallinity of the films: a) $\mathrm{Mn}_{55} \mathrm{Sb}_{36} \mathrm{Bi}_{9}$; b) $\mathrm{Co}_{45} \mathrm{Pt}_{55}$

substrates. The thickness of the MnSbBi and CoPt films ranged between 50 and $70 \mathrm{~nm}$. In this work $\mathrm{Sb} / \mathrm{Mn} / \mathrm{Bi}$ multilayers with thin $(<10 \mathrm{~nm}) \mathrm{Sb}$ seed layers were deposited on substrates for high coercivity, high remanent squareness and high perpendicular anisotropy. The films were annealed at $350^{\circ} \mathrm{C}$ in a vacuum of less than $5 \cdot 10^{-6}$ Torr. After annealing the structural properties of the $\mathrm{MnSbBi}$ films have been characterized by X-ray diffraction analysis (XRD) and high-resolution transmission electron microscopy. These measurements gave an average grain size of approximately 20-40 $\mathrm{nm}$ for $\mathrm{MnSbBi}$ films and less than $60 \mathrm{~nm}$ for $\mathrm{Co}_{\mathrm{x}} \mathrm{Pt}_{1-\mathrm{x}}$ nanocrystalline films (Figs $1 \mathrm{a}, \mathrm{b}$ ).

Optical properties of the samples were measured by spectroscopic ellipsometry in the $1.3-4.0 \mathrm{eV}$ phonon energy region at a fixed angle of incidence $\left(\varphi_{0}\right)$ equal to $72^{\circ}$. The spectro-ellipsometer is a polarizer-sample-analyzer system with fixed polarizer azimuth equal to $45^{\circ}$. Through measurement of the complex reflectance ratio $\rho\left(=r_{p} / r_{s}\right)$ of the $p$ - (parallel) and $s$-(perpendicular) field components of the light beam, defined with respect to the plane of incidence of the sample, the complex dielectric function can be obtained from the equation

$\tilde{n}=n-i k=\left[\sin ^{2} \varphi_{0}+\sin ^{2} \varphi_{0} \tan ^{2} \varphi_{0} \frac{(1-\rho)^{2}}{(1+\rho)^{2}}\right]^{1 / 2}$ by using a two-phase model (air and sample) [4,5]. The corresponding optical constant $\mathrm{n}$ and $\mathrm{k}$, as well as the dielectric functions $\varepsilon_{1}=n^{2}-k^{2}$ and $\varepsilon_{2}=2 n k$ were calculated where $\varepsilon_{1}$ and $\varepsilon_{2}$ are the real and imaginary parts of the complex dielectric constant $\varepsilon$, respectively. The diagonal component $\sigma_{x x}=\sigma_{1 x x}+i \sigma_{2 x x}$ and the offdiagonal component $\tilde{\sigma}_{x y}=\sigma_{1 x j}+i \sigma_{2 x y}$ of the conductivity tensor can be given as follows $[5,6]$ :

$$
\begin{aligned}
& \sigma_{1 x y}=(\omega / 4 \pi)\left[B \theta_{K}+A \varepsilon_{K}\right], \\
& \sigma_{2 x y}=(\omega / 4 \pi)\left[A \theta_{K}-B \varepsilon_{K}\right], \\
& \sigma_{1 x x}=(\omega / 4 \pi)\left[\frac{n B-k A}{n^{2}+k^{2}}\right], \text { and } \\
& \sigma_{2 x x}=(\omega / 4 \pi)\left[\frac{n A+k B}{n^{2}+k^{2}}\right] .
\end{aligned}
$$

The real part of diagonal component of the conductivity tensor, $\sigma_{1 x x}$, that is the optical conductivity, can be also described by the relationship $\sigma(\hbar \omega)=\mathrm{e}_{2} \mathrm{w} / 2$ (where $\mathrm{w}$ is the angular frequency of lightand, $\hbar$ is the Plank constant divided by $2 \pi$ ) at photon energy $\hbar \omega$. The spectral dependence of the optical conductivity for the samples under an external magnetic field $H=7 \mathrm{kOe}$ in the polar Kerr configuration was measured at room temperature for the same spectral region. 


\section{V.G. Kravets et al.: Optical and magneto-optical properties...}
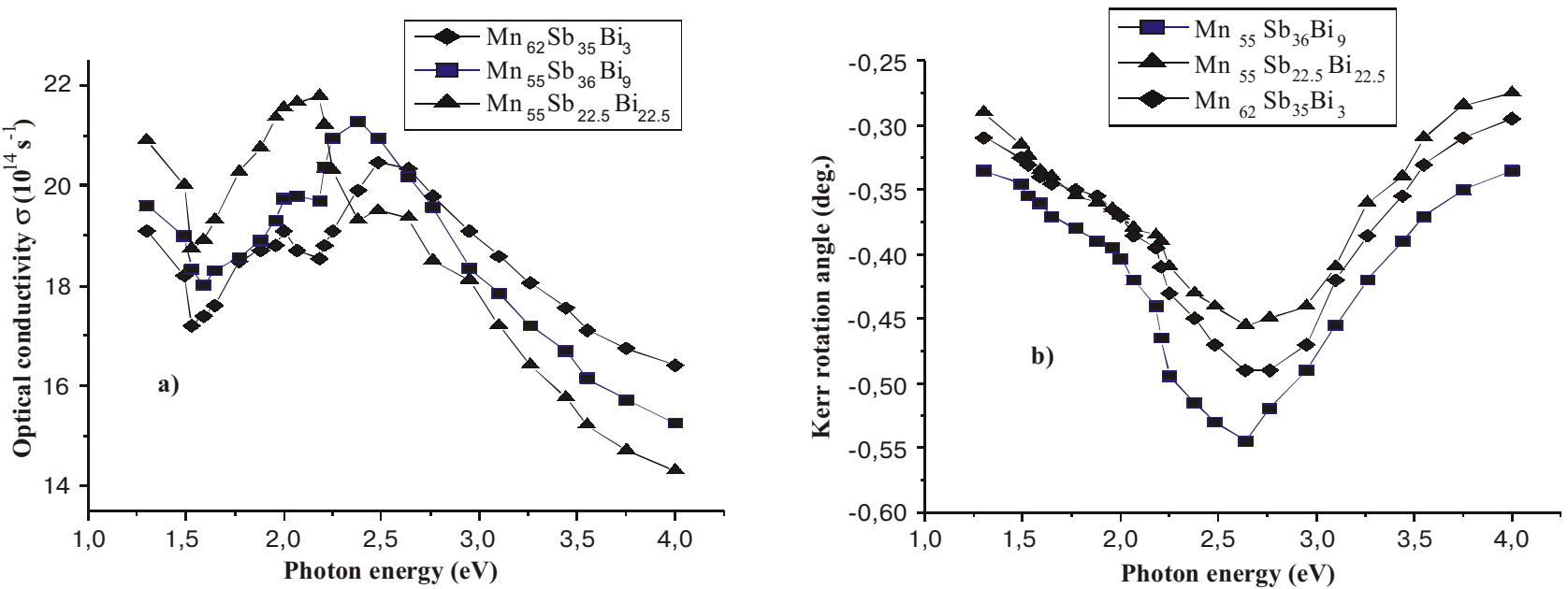

Fig. 2. a) Optical conductivity spectra $\sigma(\hbar \omega)$ for the MnSbBi nanocrystalline films; b) the polar Kerr rotation spectra for the MnSbBi nanocrystalline films

In Fig. 2a the measured absorptive parts of the diagonal optical conductivity s(hw) for Mn-Sb-Bi films are presented. At a first glance, all spectra exhibit a double peak structure with maxima around 2 and $2.6 \mathrm{eV}$. The $\sigma(\eta \omega)$ spectrum magnitude increases systematically with an increase of Bi concentration between 1.3 and $2.5 \mathrm{eV}$. With increasing Bi content the maxima at 1.9-2.1 eV increases and at $2.5-2.7 \mathrm{eV}$ decreases.

Fig. 2b show spectra of the Kerr rotation $\theta_{k}$ of Mn$\mathrm{Sb}$-Bi films. $\theta_{\mathrm{k}}$ rotation angle has been measured from the film side. It should be mentioned here that for the $\mathrm{Mn}_{55} \mathrm{Sb}_{36} \mathrm{Bi}_{9}$ film $\theta_{\mathrm{k}}$ increases from -0.35 at $4 \mathrm{eV}$ with increasing wavelength and takes maximum of $0.57 \mathrm{deg}$. at around $2.7 \mathrm{eV}$, with further increasing wavelengths, $\theta_{\mathrm{k}}$ decreases to $-0.34 \mathrm{deg}$. at $1.3 \mathrm{eV}$. The photon energy, where $\mathrm{q}_{\mathrm{k}}$ shows maximum, $\mathrm{hw}_{\max }$ is about 2.65 $\mathrm{eV}\left(\theta_{\mathrm{k}} \sim-0.43 \mathrm{deg}\right.$.) for the $\mathrm{Mn}_{55} \mathrm{Sb}_{22 . .5} \mathrm{Bi}_{22.5}$ film, $2.7 \mathrm{eV}$ $\left(\theta_{\mathrm{k}} \sim-0.5\right.$ deg.) for $\mathrm{Mn}_{62} \mathrm{Sb}_{35} \mathrm{Bi}_{3}$ film, respectively. We can see that with increasing concentration of $\mathrm{Bi}$ atoms in $\mathrm{MnSbBi}$ films Kerr rotation growths for $\mathrm{x}=3$ and 9 at. $\%$ in comparison to $\mathrm{MnSb}$ films, and after reduces by approximately $0.15 \mathrm{deg}$.

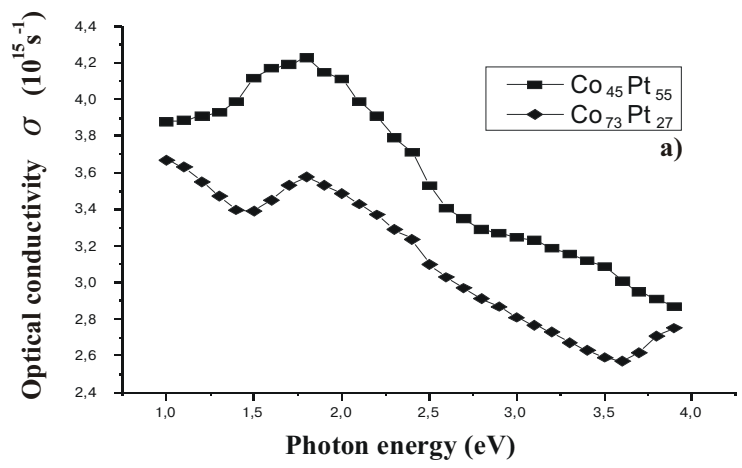

The optical conductivity spectra, $\sigma(\eta \omega)$, for the $\mathrm{Co}_{\mathrm{x}} \mathrm{Pt}_{1-}$ $x$ nanocrystalline films have been determined and are shown in Fig. 3a. The magnitude of the $\sigma(\eta \omega)$ for $\mathrm{Co}_{45} \mathrm{Pt}_{55}$ and $\mathrm{Co}_{73} \mathrm{Pt}_{27}$ nanocrystalline films is strongly enhanced in the energy region $1.5-3 \mathrm{eV}$. The features in the optical spectra of Co-Pt films are located near $1.8 \mathrm{eV}$. The optical conductivity for both films gradually increases with decreasing photon energy. It should be noticed here that $\sigma(\eta \omega)$ for the Pt-rich films is larger than the Co-rich films in the IR and visible spectral region.

In Fig. $3 b$ the evolution of the polar Kerr rotation, $\theta_{\mathrm{K}}$, spectra of those $\mathrm{Co}_{\mathrm{x}} \mathrm{Pt}_{1-\mathrm{x}}$ nanocrystalline films that exhibit magnetic ordering at the room temperature is presented. The spectra display two features. There is a prominent broad negative peak in the IR range and strongly enhancement in UV region. The characteristic value near $4 \mathrm{eV}$ in the $\theta_{\mathrm{K}}$ spectrum for $\mathrm{Co}_{\mathrm{X}} \mathrm{Pt}_{1-\mathrm{x}}$ films increases from -0.4 up to $-0.5 \mathrm{deg}$. When the Co concentration changes from $x=0.45$ to $x=0.73$ at $\%$. A relatively strong change of the $\theta_{\mathrm{K}}$ spectra in the IR region is observed and an appearing peak correlates with the peak at $1.4 \mathrm{eV}$ in $\mathrm{Co}$.

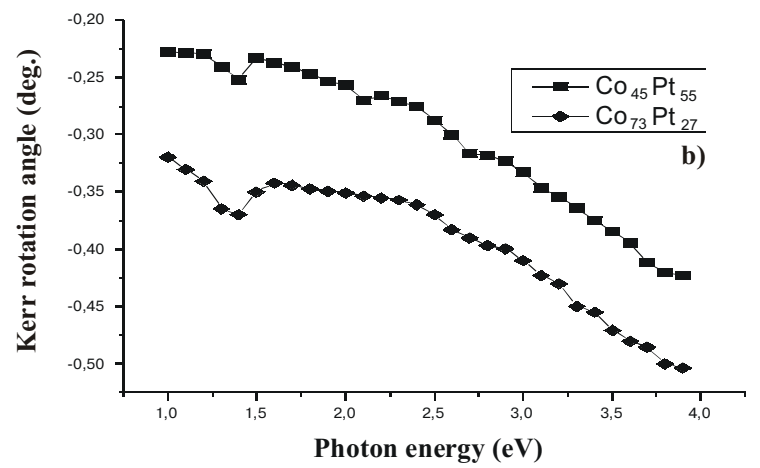

Fig. 3. a) Optical conductivity spectra $\sigma(\hbar \omega)$ for the Co-Pt nanocrystalline films; b) the polar Kerr rotation spectra for the Co-Pt nanocrystalline films 
The observed strong decrease in the $\theta_{\mathrm{K}}$ magnitude of the $\mathrm{Co}_{\mathrm{x}} \mathrm{Pt}_{1-\mathrm{x}}$ nanocrystalline films with the decrease of $\mathrm{Co}$ content is due to a drop in magnetization and reflects the Curie temperature reduction.

\section{Discussion}

It is known that magneto-optical effects arise generally from both interband transitions of valence electrons in valence bands and intraband transitions of free electrons in conduction bands. In infrared and visual regions, the contribution of free electrons to $\sigma_{2 x y}$ is proportional to $(\omega \tau)^{-1}$, where $\tau$ is the relaxation time [2,7]. It is clear that the valance band of $\mathrm{MnSb}$ and $\mathrm{MnBi}$ are derived from the $3 \mathrm{~d}$ and $4 \mathrm{~s}$ electrons of the $\mathrm{Mn}$, and the $\mathrm{s}$ and $\mathrm{p}$ electrons of the $\mathrm{Sb}$ the $\mathrm{Bi}[8,9]$. To associate the electronic structure with the magneto-optical properties, we consider the influence of the hybridization between the Mn and $\mathrm{Bi}(\mathrm{Sb})$ electronic states. Some possible reasons of microscopic scale may be proposed for the explanation of the large Kerr effect in $\mathrm{Mn}_{55} \mathrm{Sb}_{36} \mathrm{Bi}_{9}$ films: the increase of the Mn3d-Bi6p transition [10]; the change of the density of states near the Fermi level [8]. In order to analyse the density of states, the modelling of the electronic structure may be proposed to make by means of the superposition of Bi levels on $\mathrm{MnSb}$ band structure. N(E) of $\mathrm{MnSb}$ material for the majority-spin direction ( $\uparrow$ ) $3 \mathrm{~d}$ electrons shows a peaks around 2.5 and $3.7 \mathrm{eV}$ below the Fermi level, and for minority-spin direction $(\downarrow) 3 \mathrm{~d}$ electrons, a main peak situates at about $0.5 \mathrm{eV}$ above the Fermi level and a weak peak is observed near $-3 \mathrm{eV}$. We assume that doping with $\mathrm{Bi}$ atoms (doping level 10 at. \%) into $\mathrm{MnSb}$ film should affect the mixture between $3 \mathrm{~d}(\mathrm{Mn})$ and $6 \mathrm{p}(\mathrm{Bi})$ states near Fermi level, and also the transition between these states. An increase in Bi concentration leads to a larger increase in the exchange parameters $<\mathrm{J}_{0}>$. There is a strong hybridization between the $\mathrm{Bi}$ p and Mnd-type states. This rise in the $<\mathrm{J}_{0}>$ of $\mathrm{MnSb}$ upon alloying it with $\mathrm{Bi}$ is in qualitative agreement with the observed increasing in Curie temperature $T_{c}\left(T_{c}\right.$ is proportional to $<\mathrm{J}_{0}>$ ) from 550 to $600 \mathrm{~K}$. The optical active transitions take place mainly in $\mathrm{Bi}$ and $\mathrm{Sb}$ from occupied $\mathrm{p}(\eta \omega<1.7 \mathrm{eV})$ to unoccupied Mn d states in the minority spin band. The transitions perhaps occur between the Bi 6p, Sb 5p $(\downarrow)$ states located at energies EJ $2 \mathrm{eV}$ below the Fermi level and the hybridized states of $\mathrm{p}$ and $\mathrm{d}(\downarrow)$ characters lying in the energy range $0.5-1 \mathrm{eV}$ above the Fermi level $[8,10]$.

Since a large $\theta_{\mathrm{k}}$ always accompanied with the occurrence of the hexagonal orientation texture with the easy direction (c axis) vertical to the basal plane in the Mnbase films [11]. The Kerr rotation $\theta_{\mathrm{k}}$ has obviously increased when $\mathrm{MnSbBi}(002) \mathrm{X}$-ray peaks are visible after annealing at $350{ }^{\circ}$ C. $\theta_{\mathrm{k}}$ decreases if content of $\mathrm{Bi}$ is 22.5 at. $\%$. It could be explained on experimental grounds that for $\mathrm{Mn}_{55} \mathrm{Sb}_{22 . .5} \mathrm{Bi}_{22.5}$ films the easy direction of magnetization is inclined with respect to the film plane (strong peak of the Mn-Sb-Bi (101) phase). The easy direction of magnetization in $\mathrm{Mn}_{55} \mathrm{Sb}_{22 . .5} \mathrm{Bi}_{22.5}$ films lies in the basal plane and hence the film leads to a smaller polar Kerr rotation. Doping with $\mathrm{Bi}$ atoms into $\mathrm{MnSb}$ film allows to change the easy direction of magnetization from the basal plane $\left(\mathrm{Mn}_{55} \mathrm{Sb}_{22 . .5} \mathrm{Bi}_{22.5}\right)$ to the c-axis direction $\left(\mathrm{Mn}_{55} \mathrm{Sb}_{36} \mathrm{Bi}_{9}\right)$, ensuring a large readout product of Kerr rotation angle. Kerr hysteresis loops for $\mathrm{Mn}_{55} \mathrm{Sb}_{36} \mathrm{Bi}_{9}$ nanocrystalline films confirms, that this sample has perpendicular magnetic anisotropy, and its hysteresis loop is square in the perpendicular direction (Fig. 4a).

In general, a large Kerr rotation angle for Co-Pt films originates due to large spin-orbit coupling [12,13]. In [12] it was shown that the Kerr rotation spectra of Co-Pt alloys are mainly determined by two contributions. The prominent peak at $4 \mathrm{eV}$ photon energy mostly comes from the interband transitions from occupied states within a relatively narrow energy interval $\Delta \mathrm{E}_{\mathrm{j}}$ lying at $-3.4 \mathrm{eV}$ below the Fermi level $\left(\Delta \mathrm{E}_{\mathrm{j}}=0.8,1.0,1.1\right.$, and $\left.1.4 \mathrm{eV}\right)$ to another narrow interval $\Delta \mathrm{E}_{\mathrm{f}}$ just above the Fermi level $\left(\Delta \mathrm{E}_{\mathrm{f}}=0.3,0.6,0.8\right.$ and $1.0 \mathrm{eV}$ for $\mathrm{Pt}, \mathrm{CoPt}_{7}, \mathrm{CoPt}_{3}$, and $\mathrm{CoPt}$ alloys, respectively).

In order to get stable micron-sized domains, it is necessary to have a positive uniaxial magnetic anisotropy constant, $K_{u}$. A positive uniaxial magnetic anisotropy constant $K_{u}^{u}$ indicates that the easy magnetization axis lies perpendicular to the film surface, which causes high MO Kerr effect even below a saturation magnetic field.
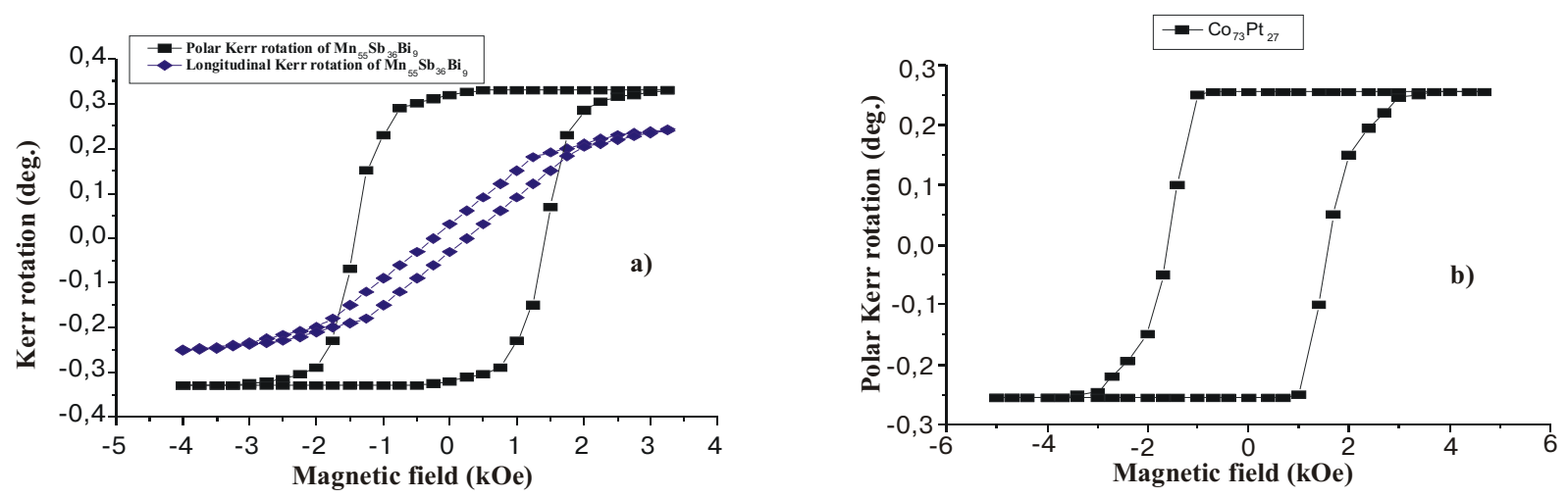

Fig. 4. a) Kerr hysteresis loop for the $\mathrm{Mn}_{55} \mathrm{Sb}_{36} \mathrm{Bi}_{9}$ nanocrystalline film as measured from the film side at $\lambda=632.8 \mathrm{~nm}$; b) $\mathrm{Kerr}$ hysteresis loop for the $\mathrm{Co}_{73} \mathrm{Pt}_{27}$ nanocrystalline film as measured from the film side at $\lambda=632.8 \mathrm{~nm}$ 


\section{V.G. Kravets et al.: Optical and magneto-optical properties...}

Domain stability requires that there be a high coercivity, $H_{c}$, and low saturation magnetization, $M_{s}$, at room temperature and at the readout temperature. Fig. $4 \mathrm{~b}$ shows the Kerr hysteresis loop of $\mathrm{Co}_{73} \mathrm{Pt}_{27}$ thin film measured at wavelength of $632.8 \mathrm{~nm}$. The loop demonstrates nearly perfect rectangular shape. This indicates that the nanocrystalline film possesses very strong perpendicular magnetic anisotropy. So easy axis of magnetization lies in the plane of perpendicular direction to the surface of film.

\section{Conclusions}

1. The optical and magneto-optical properties were determined from the results of spectroscopic ellipsometry and polar Kerr effect measurements. It was shown that $\mathrm{Mn}\left(\mathrm{Sb}_{1-\mathrm{x}} \mathrm{Bi}_{\mathrm{x}}\right)$ nanocrystalline films display new features in the magneto-optical properties and electronic structure, which are absent in $\mathrm{MnSb}$ and $\mathrm{MnBi}$ alloys.

2. A large Kerr rotation angle of about $0.57^{0}$ was observed at photon energy $\hbar \omega \approx 2.65 \mathrm{eV}$ for the $\mathrm{Mn}_{55} \mathrm{Sb}_{36} \mathrm{Bi}_{9}$ film with an average grain size of $30 \mathrm{~nm}$.

3. The changes of the exchange splitting and the electron density of states are responsible for the strong dependence of Kerr effect in $\mathrm{Mn}\left(\mathrm{Sb}_{1-\mathrm{x}} \mathrm{Bi}_{\mathrm{x}}\right)$ nanocrystalline films. 4. The optical and magneto-optical activities of the CoPt alloy thin films were measured.

The enhancement in polar Kerr rotation in the region 2$4 \mathrm{eV}$ is observed, which is characteristic for $\mathrm{Co}_{3} \mathrm{Pt}$ ordered structure.

The $\mathrm{Mn}\left(\mathrm{Sb}_{1-\mathrm{x}} \mathrm{Bi}_{\mathrm{x}}\right)$ and $\mathrm{Co}_{3} \mathrm{Pt}$ nanocrystalline films are potential candidates for future short wavelength recording MO media.

\section{References}

1. Herman J. Borg, Roel van Woudenberg, Trends in optical recording // J. Magn. Magn. Mater. 193, p. 519-525 (1999).

2. M. Mansuripur, The Physical Principles of Magneto-Optical Recording. Cambridge University, London, 1994.

3. U. Rodiger, T. Roos, P. Fumagalli, B. Hollander, G. Guntherodt, Appl. Phys. Lett. 70 p. 2604 (1997)

4. R.M.A. Azzam and N.M. Bashara, Ellipsometry and Polarized Light, North-Holland, Amsterdam, The Netherlands, 1977.

5. V.G. Kravets, A.K. Petford-Long, X. Portier, L.V. Poperenko, M. Kolesnik, J. Magn. Magn. Mater. 217, p. 129 (2000).

6. W. Reim, J. Schoenes, in: K.H.J. Bushow, E.P. Wohlfarth (Eds.), Ferromagnetic Materials, 5, North-Holland, Amsterdam, 1990.

7. P.M. Oppeneer and V. N. Antonov, in Spin-Orbit Influenced Spectroscopies of Magnetic Solids, edited by H. Ebert and G. Schutz, Springer, Heidelberg, 1996.

8. P. Ravindran, A. Delin, P. James, B. Johansson, J.M. Wills, R Ahuja, O. Eriksson, Phys. Rev. B. 59 p. 15680 (1999).

9. G.Q. Di and S. Uchiyama, Phys. Rev. B 53, p. 3327 (1996).

10. J. Kuhler and J. Kubler. J. Phys.: Condens Matter 8, p. 8681 (1996)

11. D. Weller, in: H. Ebert, G. Schstz, Spin-Orbit-Influenced Spectroscopies of Magnetic Solids, Springer, Berlin, 1996.

12. L. Uba, S. Uba, V.N. Antonov, A.N. Yaresko, R. Gontarz. Microscopic origin of the magneto-optical properties of $\mathrm{CoPt}$ alloys // Phys. Rev. B 64, p.125105 (2001).

13. G. Y. Guo and H. Ebert, J. Magn. Magn. Mater. 156, p. 173 (1996). 